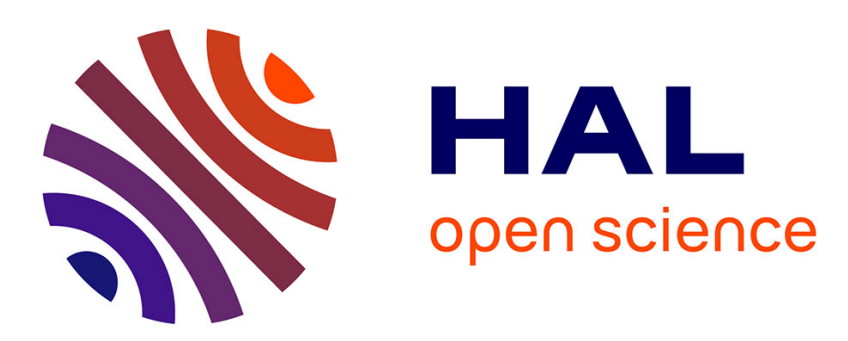

\title{
Plastic response of amorphous silicates, from atomistic simulations to experiments - A general constitutive relation
}

Gergely Molnar, Guillaume Kermouche, Etienne Barthel

\section{To cite this version:}

Gergely Molnar, Guillaume Kermouche, Etienne Barthel. Plastic response of amorphous silicates, from atomistic simulations to experiments - A general constitutive relation. Mechanics of Materials, 2017, 114, pp.1-8. 10.1016/j.mechmat.2017.07.002 . hal-02132502

\author{
HAL Id: hal-02132502 \\ https://hal.science/hal-02132502
}

Submitted on 14 Apr 2020

HAL is a multi-disciplinary open access archive for the deposit and dissemination of scientific research documents, whether they are published or not. The documents may come from teaching and research institutions in France or abroad, or from public or private research centers.
L'archive ouverte pluridisciplinaire HAL, est destinée au dépôt et à la diffusion de documents scientifiques de niveau recherche, publiés ou non, émanant des établissements d'enseignement et de recherche français ou étrangers, des laboratoires publics ou privés. 


\title{
Plastic response of amorphous silicates, from atomistic simulations to experiments - A general constitutive relation
}

\author{
Gergely Molnár ${ }^{\mathrm{b}, *}$, Guillaume Kermouche ${ }^{\mathrm{a}}$, Etienne Barthel ${ }^{\mathrm{c}, \mathrm{d}}$ \\ ${ }^{a}$ École de Mines de Saint-Etienne, Centre SMS, Laboratoire Georges Friedel \\ CNRS-UMR5307, 158 Cours Fauriel, 42023, Saint-Etienne, France \\ ${ }^{b}$ Laboratoire de Mécanique des Contacts et des Structures, Institut National des Sciences \\ Appliquées de Lyon 18-20, rue des Sciences, 69621, Villeurbanne Cedex, France \\ ${ }^{c}$ École Supérieure de Physique et de Chimie Industrielles de la Ville de Paris (ESPCI) \\ ParisTech, PSL Research University, Sciences et Ingénierie de la matière Molle, CNRS \\ UMR 7615, 10, Rue Vauquelin, F-75231 Paris Cedex 05, France \\ ${ }^{d}$ Sorbonne-Universités, UPMC Univ. Paris 06, SIMM, 10, Rue Vauquelin, F-75231 \\ Paris Cedex 05, France
}

\begin{abstract}
The continuum scale description of the plasticity of silicate glasses is a difficult task. In addition to significant amount of densification, it has been shown that, depending upon composition, there is a more or less pronounced coupling of yield stress with pressure. Moreover, the scant experimental results make it difficult it build up reliable, quantitative constitutive equations. To overcome the problem, we have recently shown that atomic scale simulations can be used to investigate the plastic response of amorphous silicates and we have proposed a generic analytical form for the constitutive relations of amorphous silicates (Molnár et al., 2016a. Acta Mat. 111, 129-137). Here we show how this generic constitutive relation can be turned into a quantitative description by calibration from micromechanics experiments. We consider the case of amorphous silica for which we have most complete sets of data.
\end{abstract}

Keywords: Silica glass, Yield criterion, Multi-scale modeling, Micro-compression, Nano-indentation

\footnotetext{
${ }^{*}$ Corresponding author

Email address: gmolnar.work@gmail.com (Gergely Molnár)
} 


\section{Introduction}

Fracture and strength of silicate glasses have been investigated with renewed vigor in the past decade. A large number of papers have dealt with the relation between composition, structure, physical and also more advanced mechanical properties - see Wondraczek et al. (2011) for a recent review of the latter. Much sparser have been the efforts towards constitutive relations which could properly account for the plastic response under both simple and more complex loadings (Kermouche et al. (2008); Keryvin et al. (2014)). However, the sound understanding of extreme mechanical response such as strength or cracking, and their composition dependence, would greatly benefit from a reliable description of the response at the continuum scale.

In fact, investigation of the plastic response of silicate glasses at the continuum scale has been limited by two major issues. First, in terms of measurements, it is difficult to obtain quantitative experimental data because large scale plastic deformations are preceded by cracking in most circumstances (Cook and Pharr (1990)). Therefore, only local (ie micron-scale) measurements can be performed. Secondly, in terms of description, it has long been known that material density may change during plastic deformation, $i e$ there is irreversible volumetric strain also called densification or compaction (Ernsberger (1968)). Therefore, we are confronted by a non conventional type of plasticity, in the sense that it does not conserve volume. Moreover, this second observation also points to the significance of hardening in the continuum scale response, which changes as the structure of the material evolves with plastic strain.

Faced with the issues of measurement difficulties, evolving structure and unconventional plasticity, alternative strategies are welcome. One of them is to resort to numerical simulations. In this field, pioneering work deduced a macroscopic yield criterion using numerical methods for bulk metallic glasses (BMGs): Schuh and Lund (2003) were able to capture the pressure dependence of the deviatoric yield strength shown experimentally Mukai et al. (2002); Zhang et al. (2003), using only atomic scale simulations. Due to the complexity of the topic, only a limited number of papers have appeared, for metallic glasses (Lund and Schuh (2003); Schuh and Lund (2003); Shimizu

et al. (2006)), nano-crystaline metals (Lund and Schuh (2004); Salehinia et al. (2014); van Swygenhoven et al. (1999)), glassy polymers (Mott et al. 
(1993); Rottler and Robbins (2001)) and other model materials (McDowell (2010); Xu et al. (2014)). Recently, we have shown that yield surfaces can be calculated by atomic scale simulations for amorphous silicates (Molnár et al. (2016a) ). However, this approach is not widespread because of some shortcomings of these simulations. With key issues such as calibration of the potential, size effects and time scale effects, atomistic simulations usually provide at most qualitative results. Here we investigate a method to turn the yield surfaces obtained from molecular statics (MS) into usable continuum scale descriptions of the material response of silicate glasses. We start from a parametric shape for the yield surface, inferred from MS, which duly reproduces the known evolutions with irreversible strain and we calibrate the parameters of the yield function from the available experimental results. Then the predictions of the resulting constitutive model are compared to other experiments and models. We also discuss the missing experimental results which would be most useful in this context.

\section{Model}

A generic analytical form for the yield criteria of silicate glasses has been evaluated numerically using Molecular Dynamics (or more precisely Molecular Statics) following the methodology reported in Molnár et al. (2016b) and Molnár et al. (2016a). In brief, through numerical experiments, different loading combinations have been applied to various systems modeling several binary glass compositions. The deformation was applied in a quasi-static manner, therefore no rate dependent effect is observable. The residual density and residual shear strain have been mapped as a function of applied stress and pressure. From these maps, a pressure dependent yield strength clearly appeared. We have therefore derived an analytical expression for the yield criterion as a function of pressure. The flow data obtained in the simulations have not been directly used for the constitutive relation but it was found that assuming associated plasticity we obtained a reasonable rendering of the computed irreversible strains (Molnár et al. (2017b); Molnár et al. (2016a)).

A typical yield surface is shown in the $p-q$ plane (Fig. 1) for a model amorphous silicat ${ }^{1}$.

\footnotetext{
${ }^{1}$ The criterion is written as a function of two invariants of the Cauchy
} 


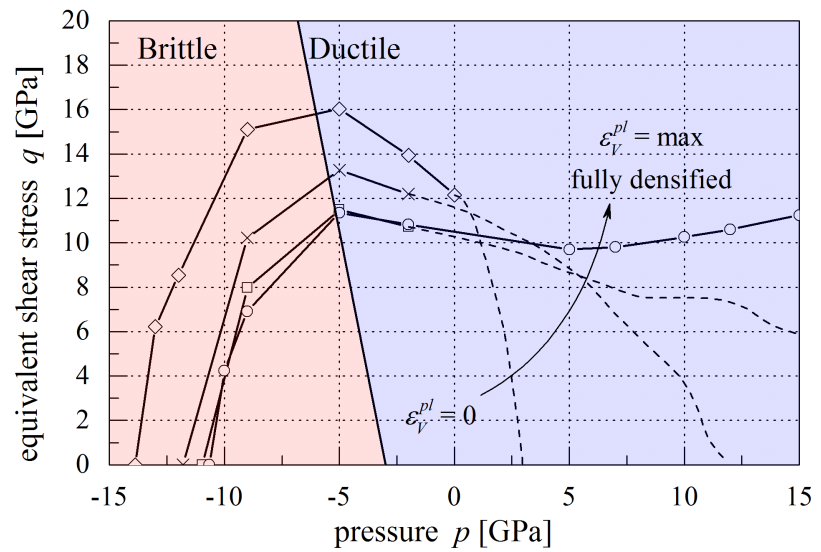

Figure 1: Shear strength as a function pressure for different material densities for silica. After Molnár et al. (2016a) and Mantisi et al. (2016).

The yield surfaces are plotted for different material densities ranging between pristine to fully densified material. In contrast to usual metal plasticity, the yield surface for the pristine, undensified material, forms a dome which reflects the strong coupling between shear and hydrostatic pressure, as observed experimentally (Mackenzie (1963); Meade and Jeanloz (1988)). Above some value of hydrostatic pressure ( $c a-5 \mathrm{GPa}$ in this case) shear strength decreases with increasing pressure. The strength curve crosses the pressure axis at the compressive (hydrostatic) strength which is the threshold for plastic deformation under pure hydrostatic pressure. Below a pressure of $c a$ -5 GPa, the shear strength decreases as pressure decreases, similarly defining the tensile (hydrostatic) strength when the curve crosses the pressure axis. As a result a peak deviatoric strength arises, equal to about 16 GPa here. As expected, this yield surface is found to evolve significantly with permanent volumetric strain $\varepsilon_{V}^{p l}$ : as density increases, the peak deviatoric strength decreases moderately, as well as the tensile (hydrostatic) strength (in absolute value), while the compressive (hydrostatic) strength increases dramatically. This strong increase of the hydrostatic compression strength reflects hard-

stress tensor: the pressure $\left(p=-\left(\sigma_{x}+\sigma_{y}+\sigma_{z}\right) / 3\right)$ and the equivalent shear stress: $q=\sqrt{\left.\left[\left(\sigma_{x}-\sigma_{y}\right)^{2}+\left(\sigma_{x}-\sigma_{z}\right)^{2}+\left(\sigma_{y}-\sigma_{z}\right)^{2}+6\left(\tau_{x y}^{2}+\tau_{x z}^{2}+\tau_{y z}^{2}\right)\right] / 2\right)}$. 
(a)

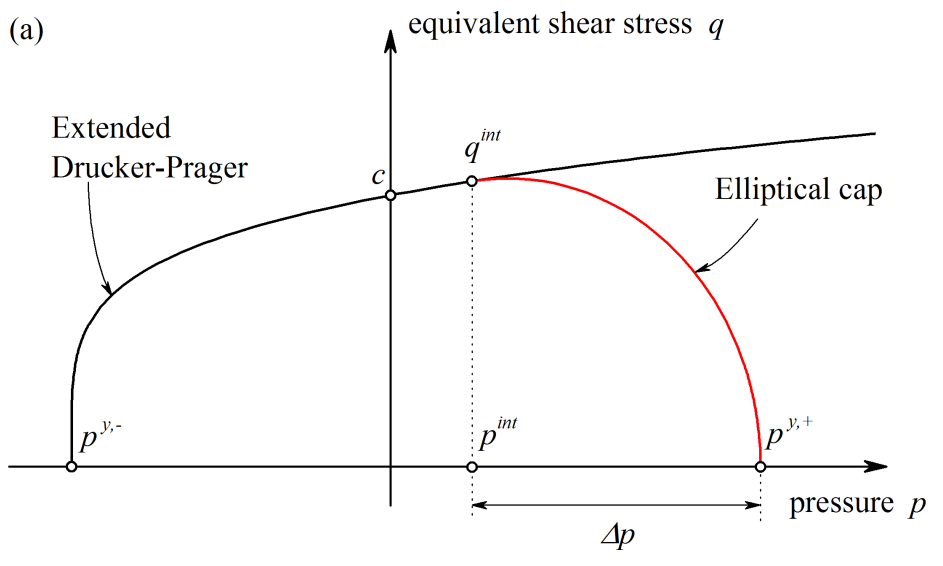

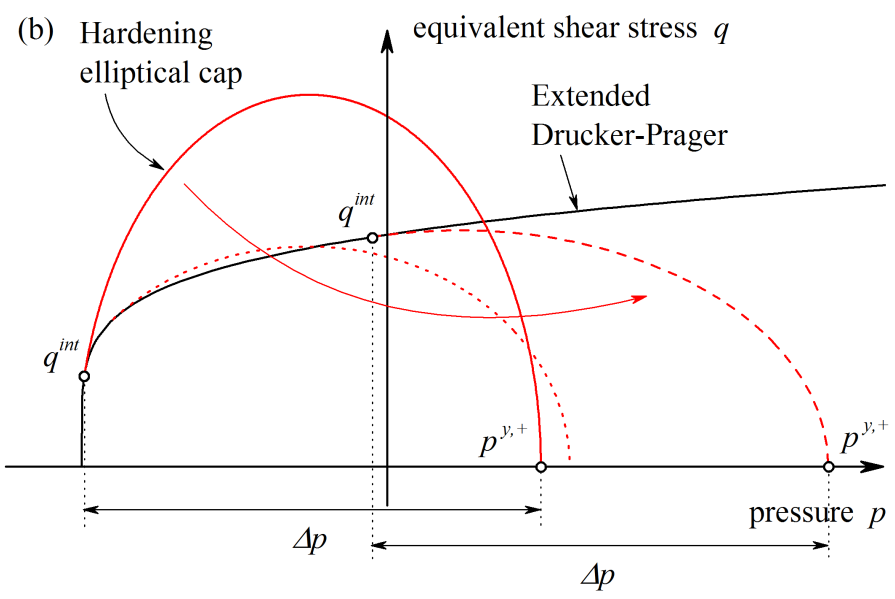

Figure 2: DP-cap yield criterion in the $p$ (pressure) - $q$ (equivalent shear stress) space, part (a) shows the parameters of the yield function. Part (b) shows how the hardening takes place and the bump disappears with densification.

ening with densification: as density saturates, the plastic response becomes shear dominated, analogous to BMGs. On the tensile side, the threshold signals instability: a homogeneous pressure state can not be maintained and macroscopic voids nucleate (Molnár et al. (2016c)). This phenomenon should be modeled with an appropriate damage model (Molnár and Gravouil (2017); Moös et al. (1999)) rather than basic computational plasticity.

Based on this evolution, we have proposed a generic shape for the yield surface (Molnár et al. (2016a)):

$$
\begin{array}{cl}
\frac{p}{p^{y,-}}+\left(\frac{q}{c}\right)^{b}-1=0 & \text { if } p \leq p^{\text {int }} \\
\left(\frac{p-h}{d}\right)^{2}+\left(\frac{q}{e}\right)^{2}-1=0 & \text { if } p^{\text {int }}<p
\end{array}
$$

In this yield surface, the tensile side of the dome is modeled by an extended Drucker-Prager model. This power law function is closed by an elliptic cap on the compression side. Under the assumption of associated plasticity, densification sets in under pure hydrostatic compression. This model will be subsequently referred to as DP-cap.

For simplicity, we have chosen to work with an associated rule. Thus equation (1) will be considered both as yield function and flow potential. This is common practice for microscopic yield criteria (Kermouche et al. (2008); Keryvin et al. (2014); Lambropoulos et al. (1996); Schuh and Lund 
(2003)).

The parameters for the DP-cap model are as follows (Fig. 2a). $p^{y,-}$ sets the tensile strength and $b$ the power law exponent of the extended DruckerPrager function: for $b=1$ we find the standard linear Drucker-Prager model while curvature increases with $b$ (Fig. 3a). Parameter $c$ sets the cohesion (Fig. 3b). The three parameters of the elliptic cap $d$, $e$ and $h$ are set by the compressive strength $p^{y,+}$ and the requirement that the two component functions meet smoothly at some pressure $p^{\text {int }}$ (Fig. 2a). Details on the governing equations can be found in our previous work Molnár et al. (2016a). As a result, the following equations can be used:

$$
\begin{gathered}
d=\frac{\Delta p^{2}+b \cdot \Delta p\left(p^{y,+}-\Delta p-p^{y,-}\right)}{2 \Delta p+b \cdot\left(p^{y,+}-\Delta p-p^{y,-}\right)}, \\
e=\frac{c\left[\Delta p+b\left(p^{y,+}-\Delta p-p^{y,-}\right)\right]}{\sqrt{p^{y,-} \cdot b\left[2 \Delta p+b\left(p^{y,+}-\Delta p-p^{y,-}\right)\right]}\left(\frac{p^{y,+}-\Delta p-p^{y,-}}{p^{y,-}}\right)^{\frac{b-2}{2 b}}}, \\
h=p^{y,+}-d .
\end{gathered}
$$

Within this framework, two simple additional features are introduced to model the complex evolution shown in Fig. 1. First the strength in compression $p^{y,+}$ increases with densification as measured experimentally. Therefore irreversible volumetric strain $\varepsilon_{V}^{p l}$ is used as an internal variable, as is standard in some recent constitutive relations for amorphous silica (Kermouche et al. (2008); Keryvin et al. (2014)). As a result, when density saturates, the cap slides to infinity and the yield function evolves smoothly into a standard BMG-like behavior (Fig. 2b). Second, we observe that the choice of pressure $p^{\text {int }}$ at which Drucker-Prager and elliptic functions meet has a strong impact on the overall shape of the yield function because it sets the slope of the ellipse on the tension side. As a result, if $p^{\text {int }}$ is close to the tensile strength $p^{y,-}$, then the elliptic cap will be prominent. If $p^{i n t}$ is far away from $p^{y,-}$, then the elliptic cap is fully included inside the Drucker-Prager function which it simply closes off on the compressive side. An interesting feature is that, although the evolution shown in Fig. 1 is complicated, with a decreasing bump with increasing density, it can be modeled very simply within the DP-cap model if we define $p^{i n t}=p^{y,+}\left(\varepsilon_{V}^{p l}\right)-\Delta p$ where $\Delta p$ is a constant. Then $p^{\text {int }}$ increases with $p^{y,+}$ as density increases and if $p^{\text {int }}$ is initially close 
a) effect of parameter $b$

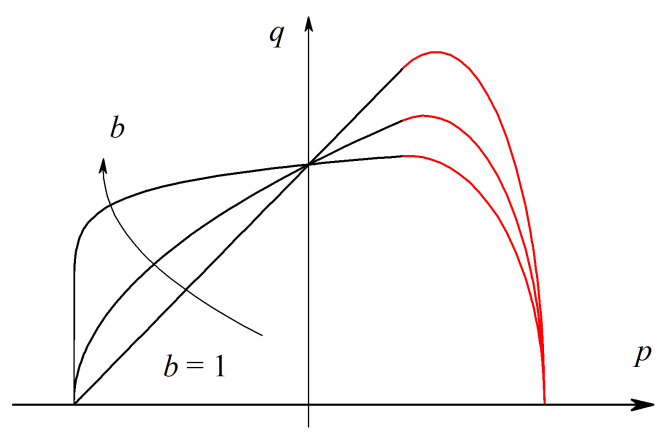

b) effect of parameter $c$

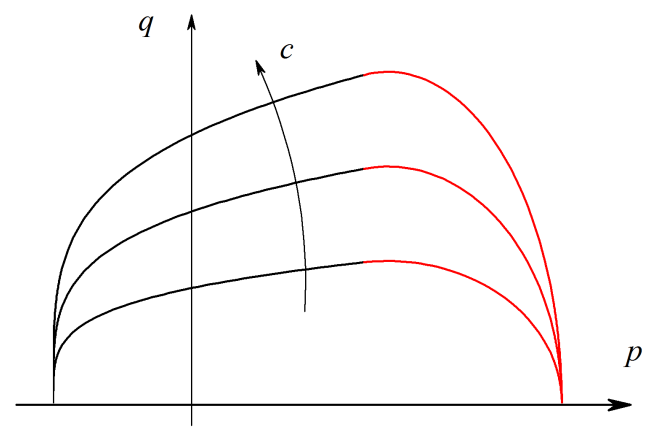

Figure 3: Effect of parameters $b$ and $c$ on the DP-cap yield criterion.

to $p^{y,-}$, the initially prominent bump will decrease to finally blend into the Drucker-Prager function as densification proceeds (Fig. $2 \mathrm{~b}$ ).

The implementation of pressure and densification coupled shear models have been extensively studied in literature, thus only the main issues are addressed here. Let us note that such constitutive models have been used for granular materials, such as geological materials by Borja and Lee (1990), pharmaceutical powders by Wu et al. (2008) and Han et al. (2008). One essential - and perhaps misleading feature - of the present work is that it addresses pressure-dependence of bulk materials, which are not usually considered porous contrary to those mentioned above. However, some similarities can be found between the yield properties of granular and bulk materials. For instance, it has been shown by Nemat-Nasser and Okada (2001) that continuous shearing induces irreversible anisotropy and densification (Nemat-Nasser (2004)) in cohesionless sand, which was surprisingly also observed in silicate glasses using atomic scale simulations by Molnár et al. (2016a).

To implement the DP-Cap yield function in ABAQUS/Standard ABAQUS (2011)) a user defined material model scheme (UMAT) is used. Details concerning the algorithm can be found in Appendix A.

\section{Experimental calibration}

In practice, to model a given amorphous silicate, $p^{y,-}, b, c, p^{y,+}\left(\varepsilon_{V}^{p l}\right)$ and $\Delta p$ must be determined. This quantitative evaluation of the numerical 
parameters - ie the calibration of the model - can be performed by comparison between numerical trials and experimental results. To that end, we need quantitative measurements for as many different types of loadings as possible. However, as stated before, one of the major constraints is the necessity to carry out these measurements at the micron-scale.

This calibration is necessary not only because of the limitations of the potentials used in the Molecular Statics calculations but also because the calculations are done without thermal activation. The experiments are conducted at room temperature, which is well below the glass transition temperature of silica, so that temperature is not expected to play a significant role in the shape of the yield ciriterion. Therefore, it may be assumed that the form of the yield function is unchanged, however the yield strength could actually be lower due to more active plastic zones.

\subsection{Experimental data sets}

An extensive set of possible experiments is shown schematically in Fig. 4 along with an indication of the average loading path in the $p$ - $q$ plane. Among these tests, we find the well known diamond anvil cell (DAC - hydrostatic compression - 6) and indentation (4) experiments, along with more recently developed tests such as pillar (5) and sphere (3) compression. Also shown is uniaxial traction (1), a test of considerably greater difficulty (Luo et al. (2016)), and pure shear (2) which has not been attempted (or at least achieved) so far. Due to experimental difficulties, a reasonably consistent series of results are available only for amorphous silica, which is why we have attempted calibration of the DP-cap model for silica glass only.

\subsection{Calibration procedure}

The compressive strength of silica under pure hydrostatic pressure and

especially its evolution with density $p^{y,+}\left(\varepsilon_{V}^{p l}\right)$ (exp. (6) in Fig. 4 ) has been extensively studied by anvil methods, either DAC or multi-anvil devices. To take this abundant data into account we use the approximate analytical dependence proposed earlier by Keryvin et al. (2014):

$$
p^{y,+}\left(\varepsilon_{V}^{p l}\right)=\left\{\begin{array}{cc}
{\left[-\ln \left(1-\frac{\varepsilon_{V}^{p l}}{\varepsilon_{V}^{p l, m a x}}\right) \frac{1}{k}\right]^{1 / m} \cdot\left(p_{m}-p_{0}\right)+p_{0}} & \text { if } \varepsilon_{V}^{p l}>\varepsilon_{V}^{p l, \max } \\
\infty & \text { otherwise }
\end{array}\right.
$$




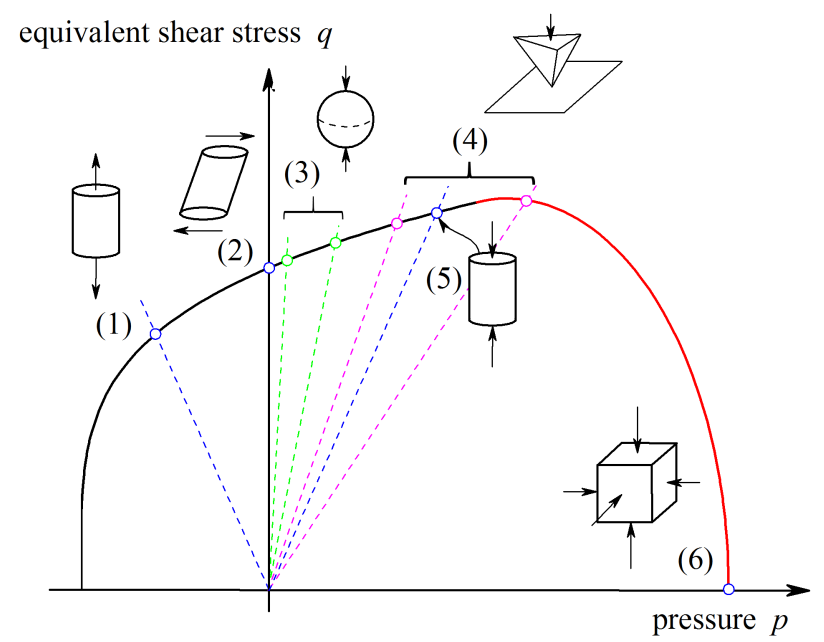

Figure 4: DP-Cap yield criterion in the space of pressure and equivalent shear stress. Different stress paths and domains are shown: 1) uniaxial tension; 2) pure shear; 3) sphere compression; 4) indentation; 5) uniaxial compression; 6) hydrostatic compression.

where $p_{0}$ is the initial yield pressure and $p_{m}, k$ and $m$ are material parameters (see Tab. 2). We chose this function to describe the hydrostatic hardening of silica, because it fits well with atomistic simulations, where almost no pure elastic domain was found (Molnár et al. (2016a)). Different experiments put the elastic limit $\left(p_{0}\right)$ of silica to different pressure values depending on their precision. For example densification is found around 7-8 GPa (Rouxel et al. (2010) ) indentation, and 9 GPa (Vandembroucq et al. (2008) ) for diamond anvil cells. Therefore to create a smooth transition between elastic and hardening domains we chose the most up to date function proposed recently by Keryvin et al. (2014). However, it needs to be strengthened that this sigmoidal function with $p_{0}=3 \mathrm{GPa}$ is only an approximation. This slight shortcoming is of no consequence for the kind of mechanical tests we are interested in here, but may lead to significant error for detailed mechanical studies near the threshold. In this case a more accurate description of the threshold is in order. The determination of $p^{y,-}$ is a bit more difficult to carry out since in this regime there is almost no data available at all. We therefore assume a reasonable value of $-5 \mathrm{GPa}$. This choice turns out to be consistent with the (very scattered) data available (Luo et al. (2016) - exp. (1) in Fig.4). 
The evaluation of $\Delta p$ is more tricky and we will comment more extensively on the impact of this parameter in the discussion. Since we know that a purely elliptic model reasonably fits indentation and pillar experiments (Kermouche et al. (2008)), we do not expect a strong bump contribution. Therefore, we assume an intermediate value for $\Delta p(7 \mathrm{GPa})$ which puts the model in the monotonically increasing strength regime (Fig. $2 \mathrm{a}$ ).

Finally, we are left with the determination of $b$ and $c$. Examination of Fig. 2 and 4 suggests that sphere compression (exp. (3)) and indentation (exp (4)) can be used for that purpose: these two types of experiments lie in a somewhat different area of the loading space where $b$ and $c$ both affect the response directly. As a result, we can expect a differentiation of these two parameters.

The elastic material properties for all models are taken according to Tab. 1. The finite element geometries for each test is summarized in Fig. 5 . Indentation tests were computed using fully integrated 3D solid elements. To save computational time the half of the actual test is modeled. Therefore, as shown in Fig. 5 a, the $y$-displacement is constrained on the middle plane. The remaining sides and the bottom of the sample were constrained completely. The load was applied on the top of the indenter using a displacement controlled method. For each indenter tip $\alpha$ and $r$ are fixed $(g=5000 \mathrm{~nm}$ and $\alpha=65.3^{\circ}$ for Berkovich), and the rest of the geometry is calculated according to: $f=\tan (\alpha) \cdot g / \sqrt{3}$ and $j=\tan (\alpha) \cdot 3 g$. The average finite element size of $200 \mathrm{~nm}$ was taken around the indenter tip.

To model sphere compression 2D axis-symmetric elements were used. The rotational symmetry is defined around axis $y$, as shown in Fig. 5b. The bottom of the silicon substrate is constrained in both $x$ and $y$ directions, while the load is applied on the top of the diamond indenter. An average of $50 \mathrm{~nm}$ finite element mesh size is used on the contact surface and $300 \mathrm{~nm}$ elsewhere.

Furthermore, to verify the calibrated parameters, micro-pillars were tested. Similarly to the sphere compression, axis-symmetric elements were used. The rotational symmetry was defined on axis $y$. The bottom and the right side of the substrate was constrained, while the load was applied on the top of the indenter. The pillar was meshed with an element size of $200 \mathrm{~nm}$.

For all contacts a 0.1 friction coefficient was used with a Penalty formulation. We have calculated the flat punch compression of a sphere to emulate available data (Romeis et al. (2015)). Based on the experimental results, we plot the maximum reaction force for a 0.927 micron punch penetration on a 

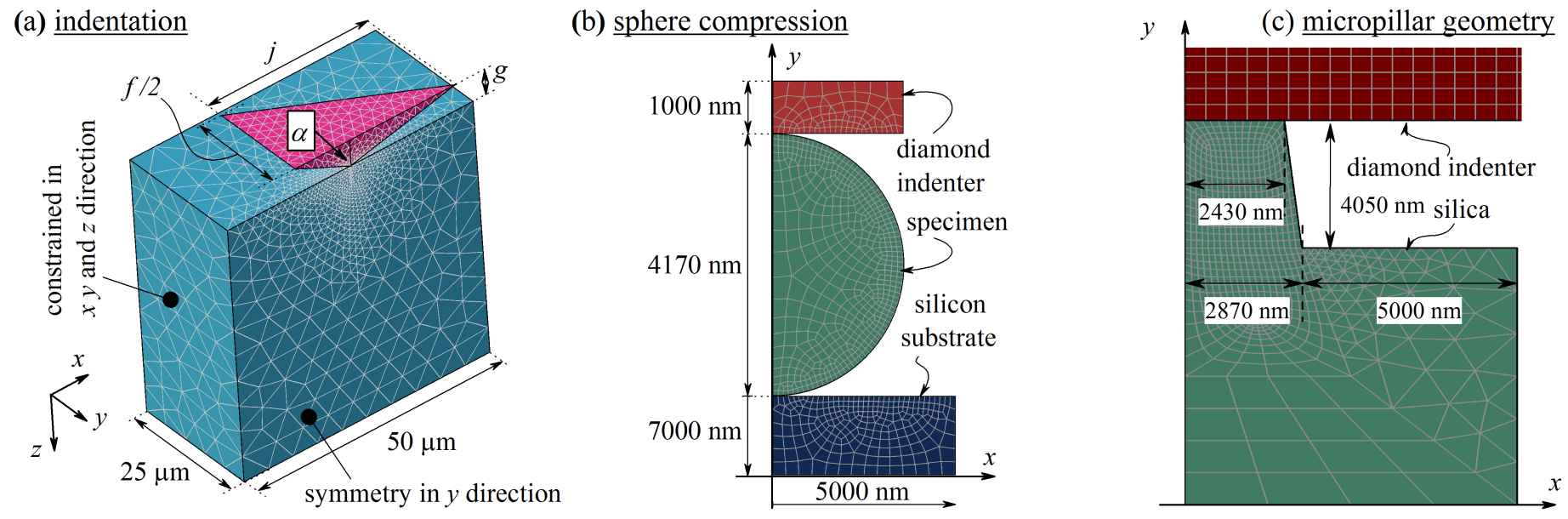

Figure 5: a) 3D finite element geometry and mesh for indentation tests. $\alpha$ shows the angle of the indenter tip. b) Axis symmetric finite element geometry for nanosphere compression. c) Axis symmetric finite element geometry for micropillar compression tests.

\begin{tabular}{lcc}
\hline Material & Young's modulus [GPa] & Poisson's ratio [-] \\
\hline Silica & 72 & 0.18 \\
Diamond (indenter) & 1000 & 0.07 \\
Silicon (substrate) & 130 & 0.22 \\
\hline
\end{tabular}

Table 1: Elastic material properties used in the finite element models.

4.17 micron silica sphere in Fig. 6b. We find that the calculated maximum reaction force is almost insensitive to the Drucker-Prager exponent $b$. This finding is in agreement with the quasi pure shear type of loading experienced in sphere compression. In contrast, the calculated maximum reaction force for a 2 micron deep Berkovich indentation (Fig. 6a) displays a clear dependence upon Drucker-Prager exponent $b$ as well as cohesion $c$ because the stress state involves more hydrostatic component. Based on the experimental values of reaction force, an optimum set of $(b, c)$ parameters can be established (Fig. 6c and Table 2).

The full loading-unloading curves calculated for the final values of the model parameters are shown in Fig. 7 for Berkovich indentation (a) and sphere compression (b). Good agreement is found with the experiments, further demonstrating that the form of the yield function is reasonable and the parameter values adequate. 

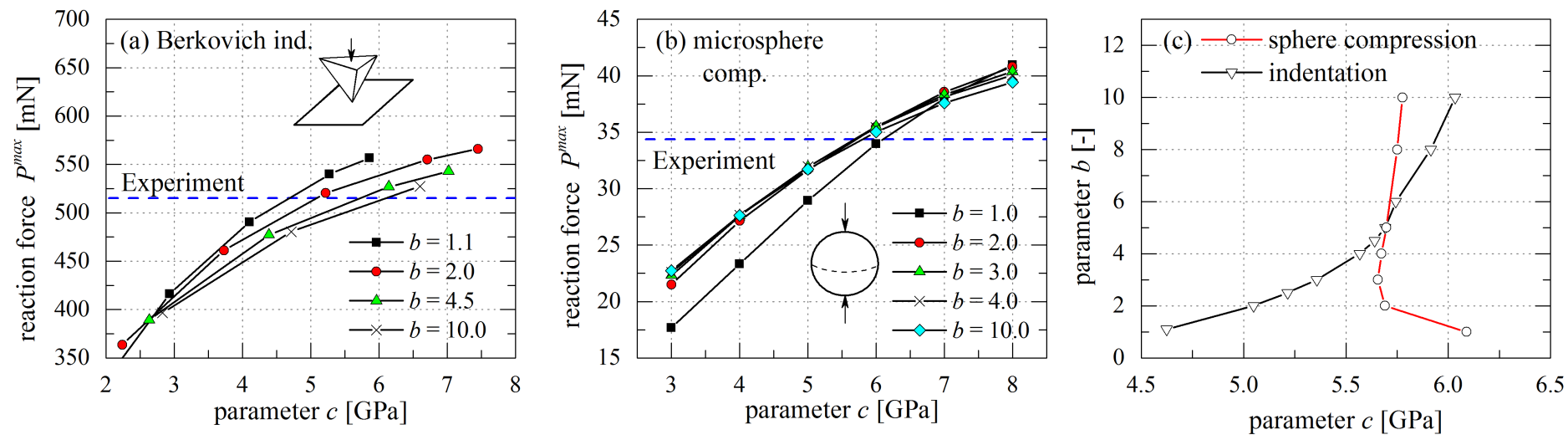

Figure 6: a) Maximum reaction force $\left(P^{\max }\right)$ for Berkovich indentation at $h^{\max }=2000 \mathrm{~nm}$ as a function of parameter $c$ for different $b$ values. Blue dashed line shows the experimental value ( $515 \mathrm{mN}$ Field et al. (2003); Iwashita and Swain (2002)). b) Maximum reaction force $\left(P^{\max }\right)$ for nanosphere compression at $h^{\max }=927 \mathrm{~nm}$ as a function of parameter $c$ for different $b$ values. Blue dashed line shows the experimental value $(34.36 \mathrm{mN}$ Romeis et al. (2015)). c) Combination of parameter $b$ and $c$ which reproduces the experimental values correctly.

\begin{tabular}{|c|c|c|c|c|}
\hline \multicolumn{5}{|c|}{ Yield properties } \\
\hline$b$ & $c$ & $\Delta p$ & $p^{y,-}$ & $p^{y,+}$ \\
\hline 5 & $5.7 \mathrm{GPa}$ & $7 \mathrm{GPa}$ & $-5 \mathrm{GPa}$ & according to eq. 5 \\
\hline \multicolumn{5}{|c|}{ Initial (undensified) cap parameters } \\
\hline$d$ & $e$ & $h$ & & \\
\hline 4.42 GPa & $5.09 \mathrm{GPa}$ & $-1.42 \mathrm{GPa}$ & & \\
\hline \multicolumn{5}{|c|}{ Positive yield pressure $\left(p^{y,+}\right)$} \\
\hline$p_{0}$ & $p_{m}$ & $k$ & $m$ & $\varepsilon_{V}^{p l, \max }$ \\
\hline $3 \mathrm{GPa}$ & $20 \mathrm{GPa}$ & 3 & 4 & -0.196 \\
\hline
\end{tabular}

Table 2: Material parameters identified and used in present work. 

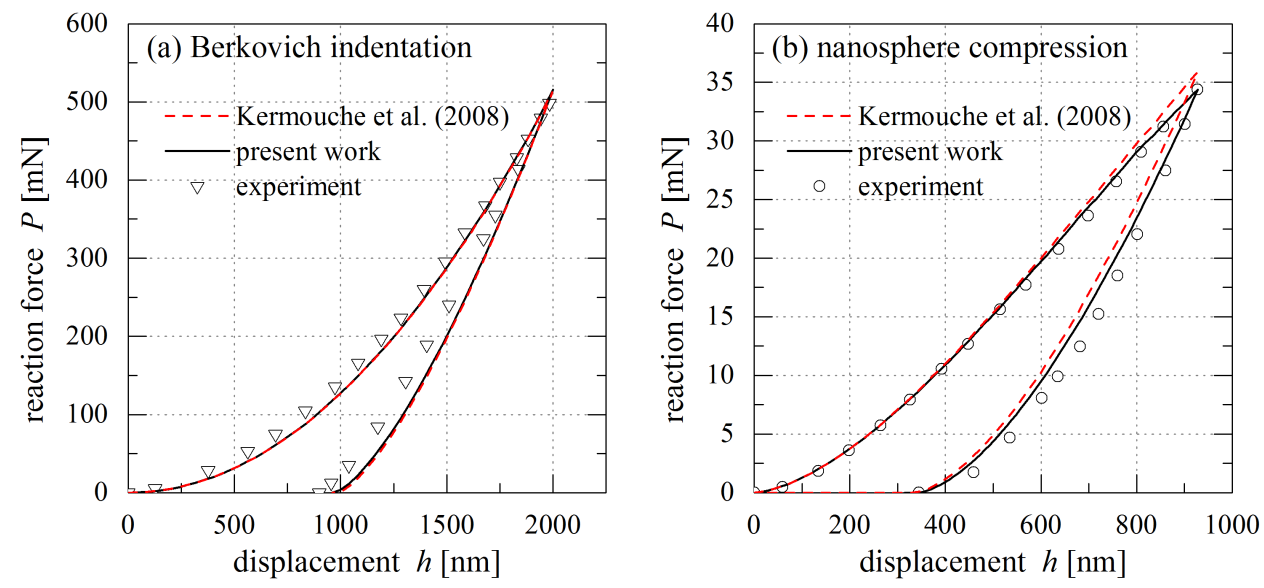

Figure 7: Reaction force as a function of displacement $(h)$. Solid black line shows the results of present work, red dashed line the results calculated using the yield criterion of Kermouche et al. (2008) and symbols represent the experimental measurements: a) Berkovich test (Field et al. (2003); Iwashita and Swain (2002)); b) Nanosphere compression $($ Romeis et al. $(2015))$.

\section{Discussion}

To assess the predictive power of the calibrated DP-cap model, we have performed additional calculations for other loading configurations. Results for indentation with sharper tips are shown in Fig. $8 \mathrm{a}$ and compared with experimental results (Field et al. (2003)). Good agreement is found although the loading curve is slightly underestimated in our calculations. We also considered pillar compression. The results are shown in Fig. 8b along with the data from Kermouche et al. (2016). A similarly good agreement is found although the predictions again slightly underestimate the loading curve.

We observe that the results of the DP-cap model do not differ significantly from the results of the standard elliptic model by Kermouche et al. (2008). When dealing with mainly compressive loadings, such as the presently available data, the models turn out very similar in effect. For silica we observe only a small pressure dependence $(b=5)$ in the DP-Cap model, while this can be much larger for other compositions. This is fully consistent with the atomistic simulations (Molnár et al. (2016a)). In the DP-cap model, the cohesion $c=5.7 \mathrm{GPa}$ ( $i e$ the strength at zero pressure) is somewhat lower than in the elliptic model (6.5 GPa). In the DP-cap model, the derivative of the 

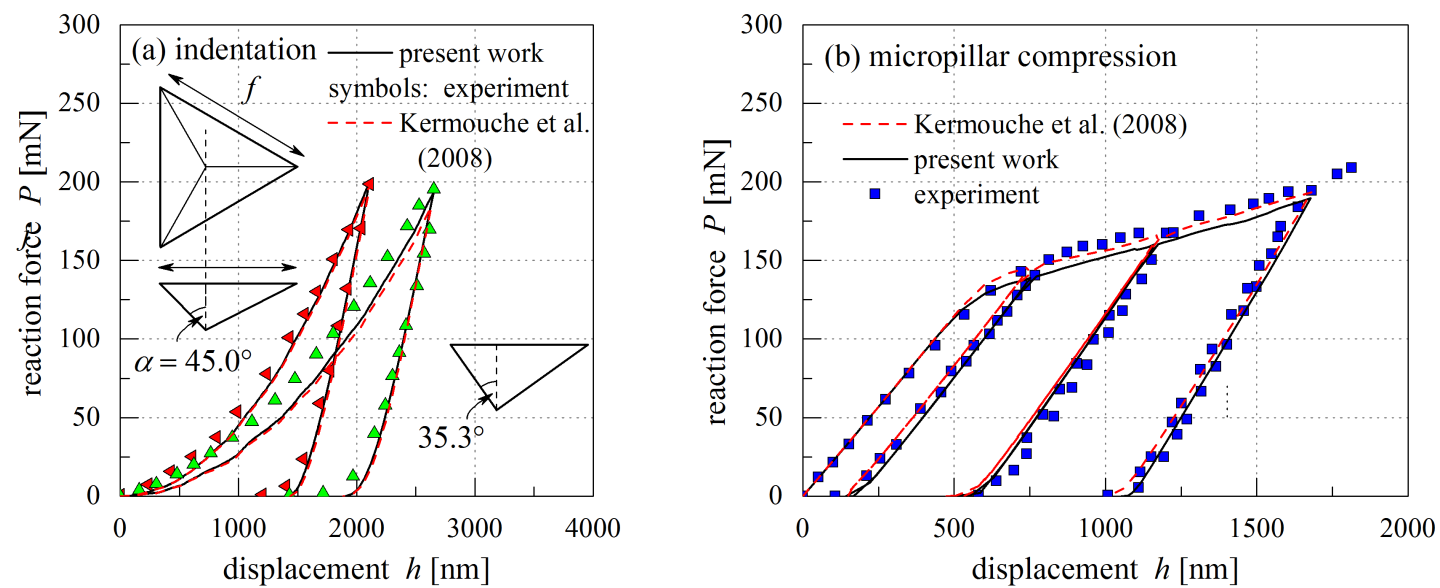

Figure 8: a) Reaction force as a function of indentation depth for $\alpha=45^{\circ}$ (red circles), cube corner (green triangle) tests (Field et al. (2003)). Black solid line shows the results of the simulation. b) Reaction force as a function of displacement for micropillar compression tests. Symbols show the experimental values (Kermouche et al. (2016)) and solid line the simulated ones.

strength with pressure is positive at zero pressure, in contrast to the elliptic model where this derivative is zero. To maintain the same average response, the cohesion is therefore lower in the DP-cap model.

Along the same line of thought, we note that in the atomic scale simulations of pure silica (Mantisi et al. (2016)), a significant bump contributes to the low hydrostatic pressure region, quite similar to Fig. 1. However we were able to model silica response with a large value of the $\Delta p$ parameter, i.e. with no bump. One reason for this discrepancy could be that strain hardening rapidly induces a reduction of the bump. More likely, the reason is that the sole datum of reaction force vs. displacement for different experimental configurations cannot discriminate between all combinations of parameter values.

A maybe more differentiating way to analyze the calculation results is to plot the spatial distribution of the stress state in the $p-q$ plane for a given experimental configuration. As an example, three different configurations are shown in Fig. 9. For indentation, a marked high pressure tail clearly appears in the stress state distribution, which is absent in pillar compression (Lacroix et al. (2012)). This tail is due to confinement by the surrounding elastic medium which strongly limits radial plastic flow, resulting in hydro- 

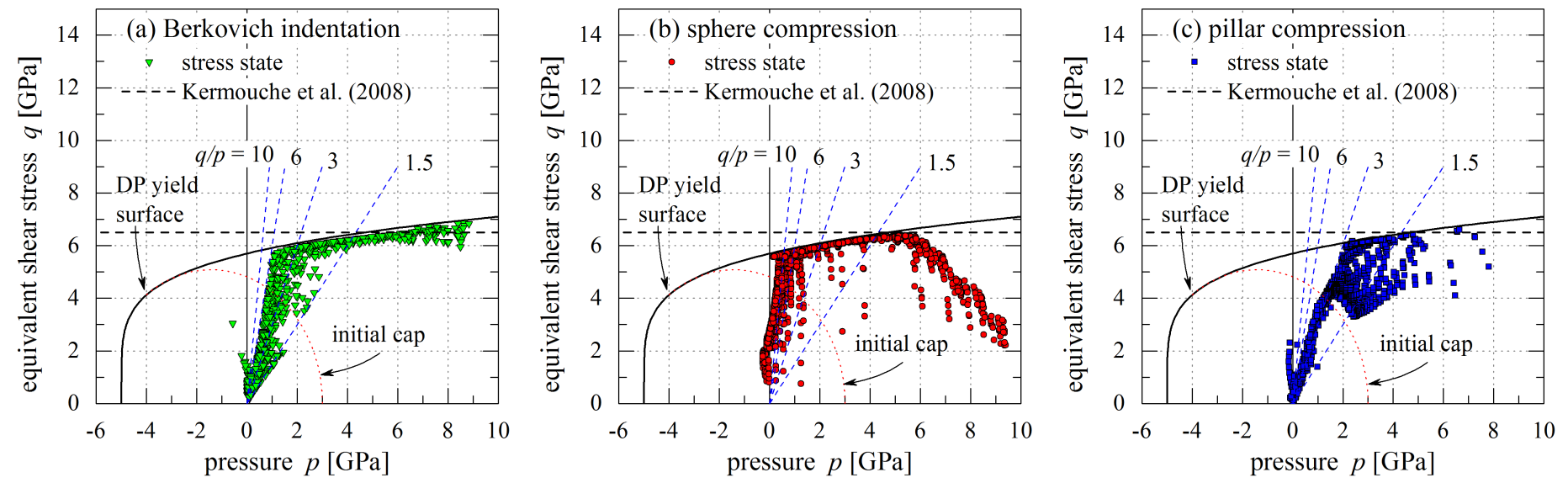

Figure 9: Stress state distribution for three types of loading at maximum penetration. (a) Berkovich indentation (b) microsphere compression (c) pillar compression. The figure also shows the yield criteria in the initial state and at full densification.

static pressure build-up and finally densification. Indeed care must be taken that tests which occupy roughly the same region in the stress state diagram (Fig. 4) may still differ markedly because of the spread in the stress states. In this respect it is interesting to note that sphere compression is somewhat intermediate between pillar compression and indentation, with some extension of the stress state into the high pressure regime. This result is not obvious from the bare inspection of Fig. 4. In fact, the sphere geometry restores some of the confinement which was lost in the pillar. From these considerations, it appears that sphere compression may be a truly fruitful pursuit in the present field.

These results suggest that beyond force displacement data, which are the result of spatial integration over a stress state distribution, full field measurements should be developed for refined definition of constitutive relations in silicate glasses. Residual strain maps are an example of this strategy (Perriot et al. (2006)). For this reason, we have investigated the impact of parameter $\Delta p$ on density distribution in Berkovich indentation. All other model parameters have been kept constant. Fig. 10 shows: a) the load displacement curves for the various values of $\Delta p$ tested; b) the density along the vertical axis $(z)$ in the symmetry plane under the indenter. We find that increasing $\Delta p$ enhances densification. This was to be expected since densification pro- 

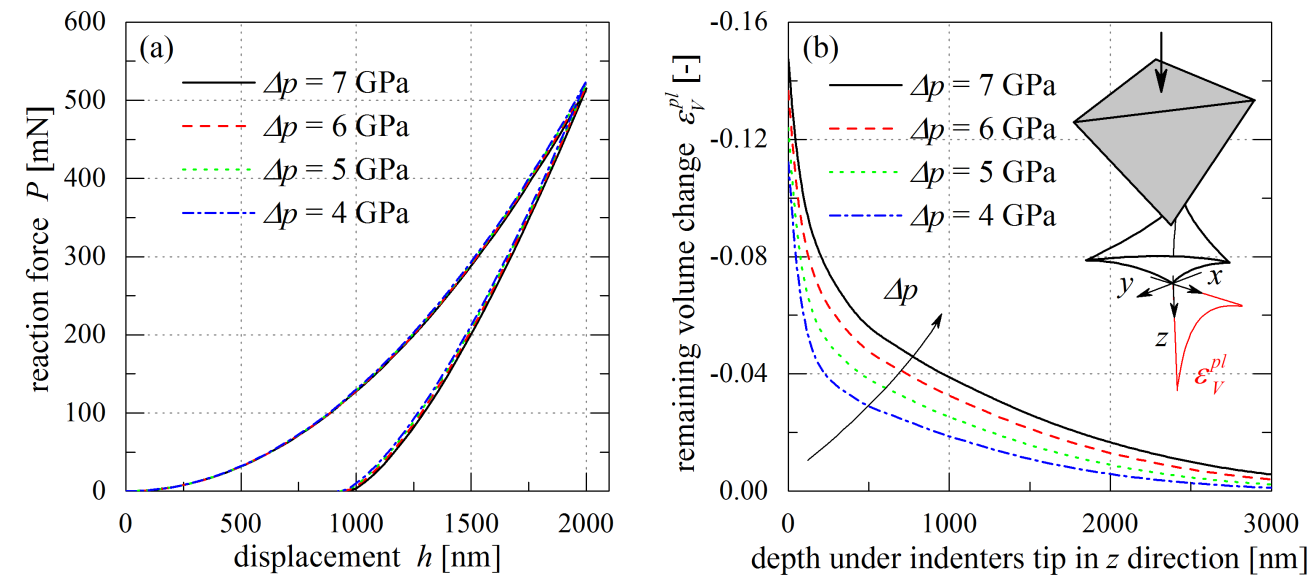

Figure 10: a) Reaction force for Berkovich indentation for different $\Delta p$ values. b) Plastic volumetric strain $\left(\varepsilon_{V}^{p l}\right)$ after unloading under the indenter tip in the silica specimen for different $\Delta p$ values.

ceeds only when the elliptic cap is reached in the yield criterion, and since for large $\Delta p$, the cap is active in a larger range of pressure. This verifies our original assumption of $\Delta p=7 \mathrm{GPa}$, showing a maximum of $\varepsilon_{V}^{p l}=15.4 \%$, which compares favorably with previous experiments (Perriot et al. (2006)). It is remarkable, however, that this variation is obtained without measurable impact on the load displacement curve.

\section{Conclusion}

Based on our previous atomic scale modeling of the plastic deformation of various silicate glasses, we have shown how a quantitative constitutive relation can be built by calibration of the analytical form to experimental results. Additional loading configurations have been calculated to test the reliability of the model. This generic form for constitutive relations of amorphous silicates is fully consistent with more specific phenomenological constitutive models proposed recently to model amorphous silica. It also provides relevant criteria for a larger variety of silicate glasses, with a well established physical basis. We have also shown that we can identify the constitutive parameters using a small set of micromechanical experiments which have been developed recently. Although the full set of data is only available for silica presently, it can relatively easily be extended to other silicate glasses in a 
near-future. Due to the increasing complexity of the constitutive models, we have also emphasized that the simpler datum of load displacement curves under various relevant loadings must be complemented by richer data sets such as strain distribution measurements. Our work also points out that experiments in the tensile regime, which are very difficult, would be most valuable at present, and that even in the case of silicate glasses ( $i e$ with modifiers) the paucity of micromechanics experiments strongly limits the accuracy of the possible descriptions. Finally, several interesting issues have not been considered here. For example, a detailed analysis of the flow rule as derived from the MD results would be a challenging increment to the present method. In a different direction, softening and the formation of shear bands would deserve specific developments as well.

\section{Acknowledgment}

This work was supported by the French Research National Agency program MultiSil (ANR-13-BS09-0012).

\section{References}

ABAQUS, 2011. ABAQUS Documentation. Dassault Systemes, Providence, RI, USA.

Borja, R. I., Lee, S. R., 1990. Cam-clay plasticity, part 1: Implicit integration of elasto-plastic constitutive relations. Computer Methods in Applied Mechanics and Engineering 78 (1), $49-72$.

Cook, R. F., Pharr, G. M., 1990. Direct observation and analysis of indentation cracking in glasses and ceramics. Journal of the American Ceramic Society 73 (4), 787-817.

de Souza-Neto, E. A., Peric, D., Owen, D. R. J., 2008. Computational Methods for Plasticity: Theory and Applications. John Wiley and Sons.

Ernsberger, F. M., 1968. Role of densification in deformation of glasses under point loading. Journal of the American Ceramic Society 51 (10), 545 - 547.

Field, J. S., Swain, M. V., Dukino, R. D., 2003. Determination of fracture toughness from the extra penetration produced by indentation-induced pop-in. Journal of Materials Research 18, 1412-1419. 
Han, L., Elliott, J., Bentham, A., Mills, A., Amidon, G., Hancock, B., 2008. A modified drucker-prager cap model for die compaction simulation of pharmaceutical powders. International Journal of Solids and Structures 45 (10), $3088-3106$.

Iwashita, N., Swain, M. V., 2002. Elasto-plastic deformation of silica glass and glassy carbons with different indenters. Philosophical Magazine A 82 (10), 2199-2205.

Kermouche, G., Barthel, E., Vandembroucq, D., Dubujet, P., 2008. Mechanical modelling of indentation-induced densification in amorphous silica. Acta Materialia 56 (13), $3222-3228$.

Kermouche, G., Guillonneau, G., Michler, J., Teisseire, J., Barthel, E., 2016. Perfectly plastic flow in silica glass. Acta Materialia 114, $146-153$.

Keryvin, V., Meng, J.-X., Gicquel, S., Guin, J.-P., Charleux, L., Sanglebœuf, J.-C., Pilvin, P., Rouxel, T., Quilliec, G. L., 2014. Constitutive modeling of the densification process in silica glass under hydrostatic compression. Acta Materialia 62, 250-257.

Krabbenhøft, K., 2002. Basic computational plasticity. Technical University of Denmark.

Lacroix, R., Kermouche, G., Teisseire, J., Barthel, E., 2012. Plastic deformation and residual stresses in amorphous silica pillars under uniaxial loading. Acta Materialia 60 (15), 5555-5566.

Lambropoulos, J. C., Xu, S., Fang, T., 1996. Constitutive law for the densification of fused silica, with applications in polishing and microgrinding. Journal of the American Ceramic Society 79 (6), 1441-1452.

Lund, A. C., Schuh, C. A., 2003. Yield surface of a simulated metallic glass. Acta Materialia 51 (18), 5399 - 5411.

Lund, A. C., Schuh, C. A., 2004. The Mohr-Coulomb criterion from unit shear processes in metallic glass. Intermetallics 12 (10-11), 1159 - 1165.

Luo, J., Wang, J., Bitzek, E., Huang, J., Zheng, H., Tong, L., Yang, Q., Li, J., Mao, S., 2016. Size-dependent brittle-to-ductile transition in silica glass nanofibers. Nano Letters 16 (1), 105-113. 
Mackenzie, J. D., 1963. High-pressure effects on oxide glasses: I. densification in rigid state. Journal of the American Ceramic Society 46 (10), 461-470.

Mantisi, B., Kermouche, G., Barthel, E., Tanguy, A., 2016. Impact of pressure on plastic yield in amorphous solids with open structure. Phys. Rev. E 93, 033001.

McDowell, D., 2010. A perspective on trends in multiscale plasticity. International Journal of Plasticity 26 (9), 1280 - 1309.

Meade, C., Jeanloz, R., 1988. Effect of a coordination change on the strength of amorphous $\mathrm{SiO}_{2}$. Science 241 (4869), 1072-1074.

Molnár, G., Ganster, P., Tanguy, A., 2017b. Effect of composition and pressure on the shear strength of sodium silicate glasses: An atomic scale simulation study. Phys. Rev. E 95, 043001.

Molnár, G., Ganster, P., Tanguy, A., Barthel, E., Kermouche, G., 2016a. Densification dependent yield criteria for sodium silicate glasses - an atomistic simulation approach. Acta Materialia 111, 129 - 137.

Molnár, G., Ganster, P., Tanguy, A., Török, J., Kermouche, G., 2016c. Transition from ductile to brittle failure of sodium silicate glasses: a numerical study. MRS Advances 1 (24), 1797-1802.

Molnár, G., Ganster, P., Török, J., Tanguy, A., 2016b. Sodium effect on static mechanical behavior of md-modeled sodium silicate glasses. Journal of Non-Crystalline Solids 440, $12-25$.

Molnár, G., Gravouil, A., 2017. 2d and 3d abaqus implementation of a robust staggered phase-field solution for modeling brittle fracture. Finite Elements in Analysis and Design 130, 27 - 38.

Moös, N., Dolbow, J., Belytschko, T., 1999. A finite element method for crack growth without remeshing. International Journal for Numerical Methods in Engineering 46 (1), 131-150.

Mott, P. H., Argon, A. S., Suter, U. W., 1993. Atomistic modelling of plastic deformation of glassy polymers. Philosophical Magazine A 67 (4), 931-978. 
Mukai, T., Nieh, T., Kawamura, Y., Inoue, A., Higashi, K., 2002. Effect of strain rate on compressive behavior of a $\mathrm{Pd}_{40} \mathrm{Ni}_{40} \mathrm{P}_{20}$ bulk metallic glass. Intermetallics 10 (11-12), 1071 - 1077.

Nemat-Nasser, S., 2004. Plasticity: A Treatise On Finite Deformation of Heterogeneous Inelastic Materials. Cambridge University Press.

Nemat-Nasser, S., Okada, N., 2001. Radiographic and microscopic observation of shear bands in granular materials. Geotechnique 51 (9), 753-766.

Oñate, E., Owen, R., 2007. Computational Plasticity. Springer Netherlands.

Perriot, A., Vandembroucq, D., Barthel, E., Martinez, V., Grosvalet, L., Martinet, C., Champagnon, B., 2006. Raman microspectroscopic characterization of amorphous silica plastic behavior. Journal of the American Ceramic Society 89 (2), 596-601.

Romeis, S., Paul, J., Herre, P., de Ligny, D., Schmidt, J., Peukert, W., 2015. Local densification of a single micron sized silica sphere by uniaxial compression. Scripta Materialia 108, $84-87$.

Rottler, J., Robbins, M. O., 2001. Yield conditions for deformation of amorphous polymer glasses. Physical Review E 642, 051801.

Rouxel, T., Ji, H., Guin, J. P., Augereau, F., Rufflé, B., 2010. Indentation deformation mechanism in glass: Densification versus shear flow. Journal of Applied Physics 107 (9), 094903.

Salehinia, I., Wang, J., Bahr, D., Zbib, H., 2014. Molecular dynamics simulations of plastic deformation in $\mathrm{nb} / \mathrm{nbc}$ multilayers. International Journal of Plasticity 59, $119-132$.

Schuh, C. A., Lund, A. C., 2003. Atomistic basis for the plastic yield criterion of metallic glass. Nature Materials 2, $449-452$.

Shimizu, F., Ogata, S., Li, J., 2006. Yield point of metallic glass. Acta Materialia 54 (16), $4293-4298$.

van Swygenhoven, H., Spaczer, M., Caro, A., Farkas, D., 1999. Competing plastic deformation mechanisms in nanophase metals. Physical Review B $60,22-25$. 
Vandembroucq, D., Deschamps, T., Coussa, C., Perriot, A., Barthel, E., Champagnon, B., Martinet, C., 2008. Density hardening plasticity and mechanical ageing of silica glass under pressure: a Raman spectroscopic study. Journal of Physics: Condensed Matter 20 (48), 485221.

Wondraczek, L., Mauro, J. C., Eckert, J., Kühn, U., Horbach, J., Deubener, J., Rouxel, T., 2011. Towards ultrastrong glasses. Advanced Materials 23 (39), 4578-4586.

Wu, C.-Y., Hancock, B., Mills, A., Bentham, A., Best, S., Elliott, J., 2008. Numerical and experimental investigation of capping mechanisms during pharmaceutical tablet compaction. Powder Technology 181 (2), 121 - 129.

Xu, Y., Chen, J., Li, H., 2014. Finite hyperelastic-plastic constitutive equations for atomistic simulation of dynamic ductile fracture. International Journal of Plasticity 59, $15-29$.

Zhang, Z. F., Eckert, J., Schultz, L., 2003. Difference in compressive and tensile fracture mechanisms of $\mathrm{Zr}_{59} \mathrm{Cu}_{20} \mathrm{Al}_{10} \mathrm{Ni}_{8} \mathrm{Ti}_{3}$ bulk metallic glass. Acta Materialia 51 (4), $1167-1179$. 


\section{Appendix A. Implementation details}

We divide the criterion into three parts: the extended Drucker-Prager part $\left(p^{y,-}<p<p^{i n t}\right)$, the left side of the ellipse $\left(p^{i n t}<p<h\right.$, where $h$ is the center of the ellipse) and the right side of the ellipse $\left(h<p<p^{y,+}\right)$. In the first two parts the slope is positive, thus the plastic volume change is positive as well, whereas, if the slope is negative, the material densifies $\left(\varepsilon_{V}^{p l}<0\right.$, where $\varepsilon_{V}^{p l}$ can be calculated by taking the trace of the plastic Hencky strain tensor). According to atomistic simulations (Molnár et al. (2016a)) positive plastic volume change does not affect the positive yield pressure, therefore when the stress path reaches the left side (either the Drucker-Prager curve or the left side of the ellipse) the equivalent plastic strain $\left(\varepsilon_{V}^{p l}\right)$, controlling the hardening, remains unchanged. As a result the implementation and the return algorithm can be separated into a left (perfectly plastic) and right (hardening) part. On the left $(p<h)$ side a radial return algorithm is used both for the Drucker-Prager and the ellipse. On the other hand, the increasing positive yield pressure complicates the implementation even with an associated flow rule since the ellipse changes in a non-radial way. Therefore, to follow the changing gradient of the yield function, a Newton-Raphson iteration is applied to satisfy the following conditions:

$$
\begin{gathered}
\underline{s}=\Delta \underline{\sigma}-\underline{\underline{C}} \Delta \underline{\varepsilon}+\underline{\underline{C}} \Delta \lambda \nabla F\left(\underline{\sigma}^{A}+\Delta \underline{\sigma}\right)=\underline{0}, \\
r=F\left(\underline{\sigma}^{A}+\Delta \underline{\sigma}\right)=0 .
\end{gathered}
$$

Equation A.1 stands for the stress condition, namely that the vectorial sum of the predicted increment $(\underline{C} \Delta \underline{\varepsilon})$ and return $\left(\underline{C} \Delta \lambda \nabla F\left(\underline{\sigma}^{A}+\Delta \underline{\sigma}\right)\right)$ must be equal to the final stress increment (see Fig. A.11). Unfortunately, if the gradient of the yield surface $(\nabla F)$ is different in point $\mathrm{A}$ and point $\mathrm{B}$, this equation cannot be solved directly. Thus the yield criterion also needs to be satisfied (eq. A.2).

The equation system is solved iteratively by minimizing gradually the residues $(s, r)$. The Jacobian of the system is updated in each $j$ internal iteration step:

$$
\left[\begin{array}{cc}
\underline{\underline{I}}+\Delta \lambda \underline{\underline{C}} \nabla^{2} F & \underline{\underline{C}} \nabla F \\
\nabla \bar{F}^{T} & \partial F / \partial \lambda
\end{array}\right]_{j}\left[\begin{array}{l}
\delta \underline{\sigma}_{j+1} \\
\delta \lambda_{j+1}
\end{array}\right]=\left[\begin{array}{l}
-\underline{s}_{j} \\
-r_{j}
\end{array}\right],
$$




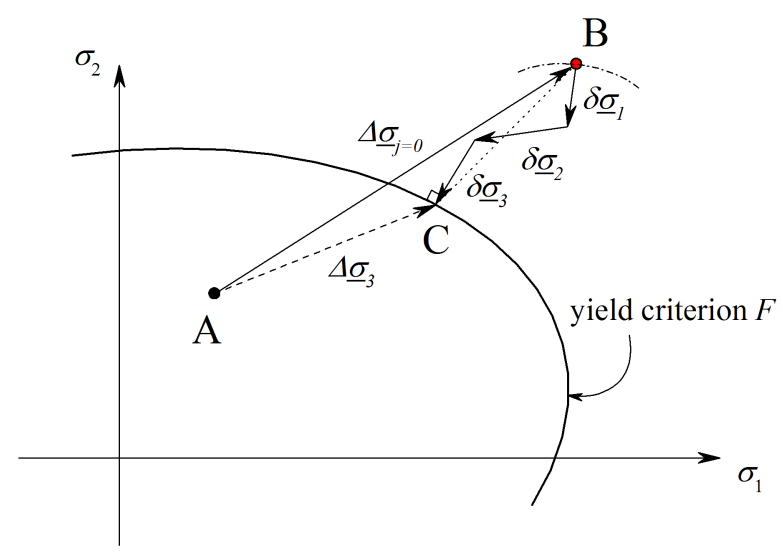

Figure A.11: Implicit non-radial return algorithm.

where $\underline{\underline{C}}$ is the elastic stiffness tensor, $\nabla F$ and $\nabla^{2} F$ are the first and second gradients of the yield surface with respect to the stress tensor at the internal step $j$ (calculated from the actual stress state $\underline{\sigma}_{j}=\underline{\sigma}^{A}+\Delta \underline{\sigma}_{j}$ ). The stress $(\Delta \underline{\sigma})$ and the plastic multiplier $(\Delta \lambda)$ increments are updated in each $j$ iterations as follows:

$$
\begin{aligned}
& \Delta \underline{\sigma}_{j+1}=\Delta \underline{\sigma}_{j}+\delta \underline{\sigma}_{j+1} \\
& \Delta \bar{\lambda}_{j+1}=\Delta \bar{\lambda}_{j}+\delta \lambda_{j+1}
\end{aligned} .
$$

The aim of the above iteration is to minimize the stress residue $(\underline{s})$ and the value of the yield function $(r)$. Therefore, after each internal step their value is updated as well. To solve the linear equation system an LU decomposition is used The plastic strain increment is defined using an associated flow rule: $d \underline{\varepsilon}_{p l}=d \lambda \nabla F$. Finally, when the residues are eliminated, all quantities are updated to calculate the tangent stiffness according to the following equation:

$$
\underline{\underline{C}}^{\tan }=\underline{\underline{C}}-\frac{\underline{\underline{C}} \nabla F \nabla F^{T} \underline{\underline{C}}}{\nabla F^{T} \underline{\underline{C}} \nabla F-\partial F / \partial \lambda} .
$$

Further information about the algorithm can be found in several textbooks discussing computational plasticity (de Souza-Neto et al. (2008); Krabbenhøft (2002); Nemat-Nasser (2004); Oñate and Owen (2007)). 